\title{
A Case of Neuromuscular Choristoma Associated with Significant Pain
}

\author{
Caroline Malcolmson, Raveena Ramphal, Daniel Keene and Donna L Johnston*
}

Division of Pediatrics, Children's Hospital of Eastern Ontario, Ottawa, Ontario, Canada

\begin{abstract}
Neuromuscular choristomas are rare tumors that usually occur in the first decade of life. They most commonly involve large nerves such as the sciatic and brachial plexus. We report a case of a 4 year old girl with a neuromuscular choristoma of the cervical region with significant pain secondary to the tumor. This is a unique region for this tumor and the persistant pain secondary to this tumor not frequently reported.
\end{abstract}

Keywords: Neuromuscular choristomas; Tumors; Cervical pain; Posterior cervical pain

\section{Introduction}

Neuromuscular Choristoma (NMC) is a rare, benign pseudo tumor that most commonly affects children in the first decade of life. NMC usually derives from a large nerve trunk, and is also known as neuromuscular hamartoma or neuromuscular rhabdomyoma or benign triton tumor [1]. It is composed of skeletal muscle and neural elements [1]. Due to its rarity, there is limited literature on this tumor type, mostly consisting of case reports. We report a case of NMC located in the left-lateral posterior cervical region of $\mathrm{C} 3-\mathrm{T} 1$ in a four year old child which caused significant persistant pain.

\section{Case Report}

A four year old female presented with a two-month history of near-constant posterior cervical pain, with decreased cervical range of motion that began at the time of an upper respiratory tract infection. She required 2-3 daily ibuprofen and acetaminophen to control the pain. The pain was exacerbated by jolting or bumping movements. The patient also reported occasional headaches, but no nausea but denied fever, weight loss or night sweats. No motor, visual, cognitive or behavioral problems were reported.

Physical exam revealed a well looking child with normal vital signs. Asymmetry of the posterior neck was noted with a slight fullness on the left compared to the right. Several café au lait spots were noted on the patient's mid-back. Examination of cervical range of motion revealed full forward flexion, however, pain on extension. Right lateral rotation was limited by 30 degrees and left lateral rotation was limited by 15 to 20 degrees. No neurological deficits were noted. Ophthalmological examination revealed normal vision with no deficits.

Bloodwork was all normal apart from an ESR that was slightly elevated at $21 \mathrm{~mm} / \mathrm{Hr}$. MRI imaging revealed a left paraspinal lesion at C5-T1 with bright T2 signal and enhancement of the vertebral bodies and lamina, and neural foramina extension at the level of the left C3 to T1. There was no spinal cord involvement with the mass and no thecal sac compression was evident. Results of bone scanning and MIBG scanning were not consistent with a malignant process. HVA and VMA were normal, CT chest was normal with no evidence of pulmonary metastases, MRI of the abdomen and pelvis was normal. Genetic results were negative for neurofibromatosis 1 .

An open biopsy and an ultrasound-guided percutaneous biopsy were performed. The samples showed changes suggestive of repair, although, no definite tumor was found and samples were negative for malignancy.

The patient nonetheless experienced worsening neck pain centered on the left side of the lower cervical spine region, combined with neuropathic pain radiating down both arms and weakness in left hand grip. The patient also began to show fatigue, as well as decreased appetite and activity. An excisional biopsy was therefore performed. At this surgery it was noted that the mass encapsulated a nerve. Following these third biopsy the patient's symptoms improved slightly. Pathological examination revealed a diagnosis of neuromuscular choristoma. Pain control remained incomplete, however, and the patient was placed on gabapentin and acupuncture therapy.

\section{Discussion}

Neuromuscular choristomas are benign tumors typically containing skeletal muscle and neural components [1]. This tumor was first reported in 1895 by Orlandi who described a case involving the sciatic nerve [2]. NMCs are also known as "benign Triton tumors" and this term refers specifically to tumors which derive from neoplastic (typically Schwann) nerve sheath cells that exhibit myogenic differentiation [3]. While the histological origin of NMCs is disputed, one theory suggests that skeletal muscle cells are trapped by nerve cells at the time of embryogenesis [4]. This is consistent with the finding that NMCs typically occur at sites of large nerve bodies such as the brachial plexus, sciatic nerve and proximal median nerve [1]. In our case, skeletal muscle cells were found in conjunction with nerve fibers at a site neural tissue. Furthermore, NMCs typically form fibrous tissue, as was seen on excision, due to degeneration of muscle and nerve cells from lack of stimulation [3].

Neuromuscular choristomas most frequently occur in the first decade of life, most often in infants and young children [1]. The size, location and degree of impingement on surrounding structures determines the disability associated with this tumor, and some children have developed striking disabilities secondary to this tumor including foot deformities [1]. There is not a significant documentation of persistent pain secondary to this tumor reported in the literature, which our patient experienced.

NMCs are isolated entities and not associated with systemic diseases such as neurofibromatosis [5]. A review of 14 cases in the literature found that these lesions had a mean size of $2.75 \mathrm{~cm}, 71 \%$

*Corresponding author: Donna Johnston, Division of Hematology/Oncology, Children's Hospital of Eastern Ontario, 401 Smyth Road, Ottawa, ON, K1H 8L1, Canada, Tel: 613-737-7600 ext 2210; Fax: 613-738-4828; E-mail: djohnston@cheo.on.ca

Received May 15, 2014; Accepted May 28, 2014; Published May 30, 2014

Citation: Malcolmson C, Ramphal R, Keene D, Johnston DL (2014) A Case of Neuromuscular Choristoma Associated with Significant Pain. J Clin Case Rep 4 367. doi:10.4172/2165-7920.1000367

Copyright: (c) 2014 Malcolmson C, et al. This is an open-access article distributed under the terms of the Creative Commons Attribution License, which permits unrestricted use, distribution, and reproduction in any medium, provided the original author and source are credited. 
Citation: Malcolmson C, Ramphal R, Keene D, Johnston DL (2014) A Case of Neuromuscular Choristoma Associated with Significant Pain. J Clin Case Rep 4: 367. doi:10.4172/2165-7920.1000367

were associated with a large nerve, and half were discovered between birth and 1 year [5]. They also found that the male-female ratio was 1:2 and recurrence was uncommon. The majority of cases of NMC are solitary tumors, but there are cases of multiple lesions [6]. The majority of patients with NMC undergo complete resection of the lesion, if possible without neurologic sequelae [7].

A recent review of the magnetic resonance imaging findings of NMC showed enlargement of the nerves with T1 and T2 signal characteristics following those of muscle [8]. This review of 6 patients found that 4 presented with pain, similar to our patient, and this is one of the only reports of the pain associated with this tumor. It does not however describe the ongoing, long term pain that our patient experienced, which makes this case unique.

The natural history of this tumor is not well understood due to the rarity of this tumor, but there is literature describing the development of fibromatosis in patients with NMC $[7,8]$. One of these articles described the development of aggressive fibromatosis in four of five patients in long term follow up after biopsy [8]. Thus, all patients with the diagnosis of NMC require careful consideration of intervention as well as close follow up once the diagnosis is established.

\section{References}

1. Kim SY, Kown HP, Kwak KD, Ahn KB (2005)Neuromuscularchoristoma of the sciatic nerve - A case report. Korean J Pathol 39:192-196.

2. Orlandi E (1895)Soprauncaso di rabdomioma del nervoischiatico. Arch Sci Med 19:113-137.

3. Mitchell A,Scheithauer BW, Ostertag H, Sepehrnia A, Sav A (1995) Neuromuscular choristoma. Am J Clin Pathol 103: 460-465.

4. Zwick DL, Livingston K, Clapp L, Kosnik E, Yates A (1989) Intracranial trigeminal nerve rhabdomyoma/choristoma in a child: a case report and discussion of possible histogenesis. Hum Pathol 20: 390-392.

5. Tiffee JC, Barnes EL (1998) Neuromuscularhamartomas of the head and neck. Arch Otolaryngol Head Neck Surg 124: 212-216.

6. Maher CO, Spinner RJ, Giannini C, Scheithauer BW, Crum BA (2002) Neuromuscular choristoma of the sciatic nerve. Case report. J Neurosurg 96: 1123-1126.

7. HÃ@bert-Blouin MN,Scheithauer BW, Amrami KK, Durham SR, Spinner R (2012) Fibromatosis: a potential sequela of neuromuscular choristoma. J Neurosurg 116: 399-408

8. Niederhauser BD, Spinner RJ, Jentoft ME, Everist BM, Matsumoto JM, et al (2013) Neuromuscular choristoma: characteristic magnetic resonance imaging findings and association with post-biopsy fibromatosis. Skeletal Radiol 42: 567577. 